\title{
Time-Resolved Studies of Ultracold Ionizing Collisions
}

\author{
Scott D. Bergeson \\ scott.bergeson@byu.edu \\ C. Orzel \\ S. Kulin \\ S. L. Rolston
}

Follow this and additional works at: https://scholarsarchive.byu.edu/facpub

Part of the Astrophysics and Astronomy Commons, and the Physics Commons

\section{Original Publication Citation}

C. Orzel, S. D. Bergeson, S. Kulin, and S. L. Rolston, "Time-resolved studies of ionizing collisions," Physical Review Letters, 8, 593-596 (1998). The original publication of this article can be found at http://link.aps.org/doi/1.113/PhysRevLett.8.593

\section{BYU ScholarsArchive Citation}

Bergeson, Scott D.; Orzel, C.; Kulin, S.; and Rolston, S. L., "Time-Resolved Studies of Ultracold lonizing Collisions" (1998). Faculty Publications. 653.

https://scholarsarchive.byu.edu/facpub/653

This Peer-Reviewed Article is brought to you for free and open access by BYU ScholarsArchive. It has been accepted for inclusion in Faculty Publications by an authorized administrator of BYU ScholarsArchive. For more information, please contact ellen_amatangelo@byu.edu. 


\title{
Time-Resolved Studies of Ultracold Ionizing Collisions
}

\author{
C. Orzel,* S. D. Bergeson, S. Kulin, and S. L. Rolston \\ National Institute of Standards and Technology, PHY A167, Gaithersburg, Maryland 20899
}

(Received 18 February 1998)

\begin{abstract}
Using $40 \mathrm{~ns}$ laser pulses, we probe the real-time dynamics of ultracold ionizing collisions in metastable xenon. We time resolve both shielding and enhancement effects, and observe the production of $\mathrm{Xe}_{2}^{+}$molecular ions through associative ionization. We estimate the rate of molecule formation in excited-state collisions, and directly measure the role of both flux enhancement and excited state survival in the collisional enhancement process. Conceptually simple theoretical models are used to predict the dynamics of the collisional shielding. [S0031-9007(98)06336-4]
\end{abstract}

PACS numbers: $34.50 . \mathrm{Rk}, 32.80 . \mathrm{Pj}, 34.50 . \mathrm{Fa}$

The study of ultracold ( $T \leq 1 \mathrm{mK}$ ) collisions between atoms has attracted a great deal of interest in recent years, in part because the long time scales involved $(t \sim$ $1 \mu \mathrm{s})$ allow for absorption and emission of photons during the collision process. Such photon scattering can increase or decrease the collision rate, allowing control of the collision process by the application of appropriately tuned laser light $[1-5]$. The low velocities and long distances involved provide an opportunity to monitor atomic collisions in real time. Using short pulses of laser light, we probe the dynamics of the collisions on a $\sim 100 \mathrm{~ns}$ time scale. We distinguish between Penning ionization $\left(\mathrm{Xe}^{*}+\mathrm{Xe}^{*} \rightarrow \mathrm{Xe}^{+}+\mathrm{Xe}+e^{-}\right)$and the formation of $\mathrm{Xe}_{2}^{+}$molecules through associative ionization $\left(\mathrm{Xe}^{*}+\mathrm{Xe}^{*} \rightarrow \mathrm{Xe}_{2}^{+}+e^{-}\right)$, and directly observe the role of excited-state survival and flux enhancement effects in collision enhancement. We also time resolve the collision shielding process, and use a simple theoretical model to predict the time behavior of this process. We note that Gensemer and Gould [6] have recently studied the time dependence of trap-loss collisions in a rubidium magnetooptic trap (MOT).

The basic physical picture of laser modification of collisions is shown in Fig. 1 and presented in detail in Ref. [7]. A pair of two-level atoms approaching each other in their ground $(S)$ states (the "ground state" here being a metastable state with enough internal energy for Penning ionization) absorbs a photon from a laser tuned near the $S \rightarrow P$ transition. The laser excites the atoms to one of the two $S+P$ molecular excited state potentials $\left[U(R)= \pm C_{3} / R^{3}\right]$ at a specific internuclear separation $R_{c}=\left(C_{3} / \hbar \Delta\right)^{1 / 3}$ (the Condon radius), determined by the detuning $\Delta=\left(\omega-\omega_{o}\right)$ from atomic resonance, and the long-range dipole-interaction strength $C_{3}$. Typical experimental values of $R_{c}$ are $\sim 1000 a_{o}$, (where $a_{o}$ is the Bohr radius) well outside the region of small internuclear separation where inelastic processes such as Penning or associative ionization occur. For blue-detuned $(\Delta>0)$ light, the atom pair is excited to the repulsive molecular state, and is prevented from reaching the inelastic collision region. This optical shielding suppresses the collision rate.
For red-detuned $(\Delta<0)$ light, the pair is excited to the attractive molecular state, and the atoms are accelerated towards one another. We then find an enhancement of the Penning ionization rate from one of two processes. Some fraction of the atom pairs thus excited will survive in the excited state long enough to reach the inner region, producing excited-state Penning ionizing collisions (EPI), which would not occur in the absence of the laser light. The remaining pairs will spontaneously decay back to the ground state before an inelastic collision occurs. They may, however, gain enough energy from acceleration on the excited potential to overcome centrifugal barriers and collide in higher angular momentum channels than their thermal energy would normally allow; this gives an enhancement of the

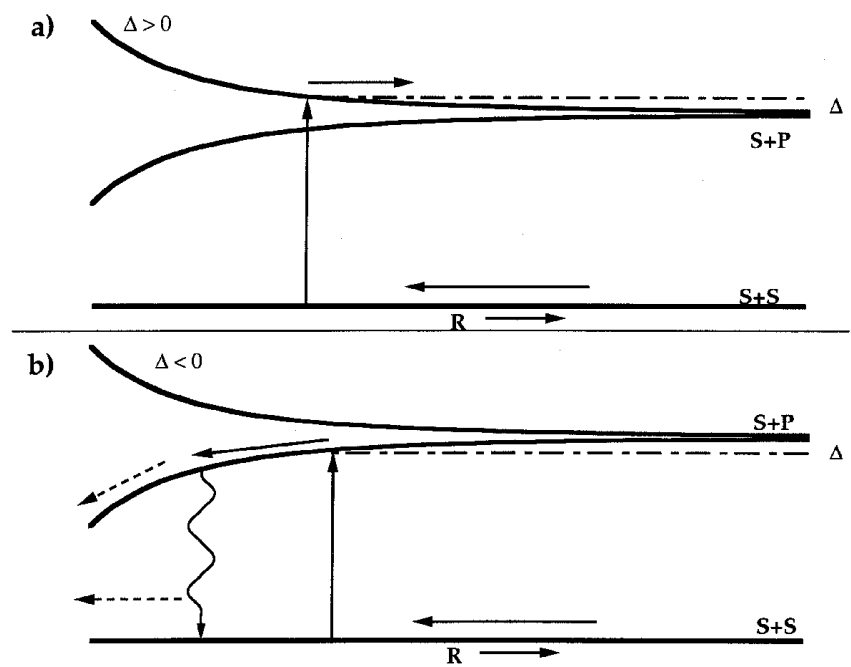

FIG. 1. Schematic of the laser-modified collision process. (a) Shielding $(\Delta>0)$ : Atoms approach on the ground $S+S$ potential and are excited by the probe laser to the repulsive $S+P$ potential. They are reflected without reaching the shortrange ionizing collision region. (b) Enhancement $(\Delta<0)$ : Atoms are excited to attractive molecular states, and accelerated together. After some time, they may decay to the ground state and continue in at higher velocity (flux enhancement), or remain on the excited state into the ionization region. 
ground-state Penning ionization (GPI) rate. This is the "flux enhancement" effect of Ref. [3], and is most important for extremely long-range excitations, where the probability of survival is low.

These processes may also enhance the rate of associative ionization [8]. The rate of ground-state associatively ionizing collisions can be increased through flux enhancement, and pairs surviving in the excited state can undergo excited-state associative ionization (EAI), colliding to form molecules. The doubly excited $(P+P)$ potential is flat at long range, and should produce no enhancement of either rate for those few pairs excited to it.

The real $\mathrm{Xe}^{*}$ situation is more complex than this two-level model. There are 20 attractive and 20 repulsive potentials stemming from the $6 s[3 / 2]_{2}+6 p[5 / 2]_{3}$ separated-atom asymptote, all of which may contribute to the shielding or enhancement. These states arise only from different arrangements of molecular orbitals, and not from atomic hyperfine structure [9]. This is a significant simplification, as there are no crossings between the potentials, and we view the $\mathrm{Xe}^{*}$ situation as an ensemble of many two-level systems, each undergoing laser excitation at a different $R_{c}$.

The present experiment consists of applying a short ( $\leq 40 \mathrm{~ns} \mathrm{rms}$ width) pulse of laser light tuned near the $882 \mathrm{~nm} 6 s[3 / 2]_{2} \rightarrow 6 p[5 / 2]_{3}$ transition to an ultracold sample of atoms in a $\mathrm{Xe}^{*} \mathrm{MOT}$. The apparatus for cooling and trapping metastable xenon is described in Ref. [10]. The slowing and trapping laser beams are chopped at a rate of $\sim 2 \mathrm{kHz}$, with an "off" period of $80 \mu \mathrm{s}$. Another laser is used to generate the probe pulse $40 \mu \mathrm{s}$ after the trapping lasers are extinguished. The collision rate is observed by monitoring the rate of ion production. Ions are drawn into and detected by a channel electron multiplier mounted $\sim 6 \mathrm{~cm}$ away, and the ion counts are recorded with a multichannel scaler, providing a histogram of ion production vs time.

The probe laser excites those atom pairs with internuclear separations near $R_{c}$, which then undergo either an enhancement or shielding process, depending upon the probe detuning, giving rise to a brief increase or decrease in the collision rate. Atom pairs at radii far from $R_{c}$ are unaffected, and provide a constant background of ionizing collisions.

Typical data for both red and blue detunings are shown in Fig. 2. The $\sim 750 \mathrm{~mW} / \mathrm{cm}^{2}$ probe pulse [11] is applied at $t=0$, and the $\sim 9 \mu$ s delay before observation of the enhancement or suppression effect reflects the time of flight to our detector. We measure this time of flight in a separate experiment (inset) using the signal from direct photoionization of $\mathrm{Xe}^{*}$ atoms in the $6 p$ state in the MOT by a $5 \mathrm{~ns}$ pulse from a $514 \mathrm{~nm}$ dye laser. This signal, peaked at 8.90(5) $\mu \mathrm{s}$ [12], also shows the resolution of our ion collection system, with an rms width of $110 \mathrm{~ns}$; convolved with the $40 \mathrm{~ns}$ pulse width, this gives us an experimental resolution of $\sim 120$ ns.
Figure 2(a) presents data for red $(\Delta<0)$ detunings. The initial large enhancement peak is due to $\mathrm{Xe}^{+}$ions, from Penning ionization in either flux-enhanced $6 s+6 s$ (GPI) or $6 s+6 p$ (EPI) collisions. The second peak is from $\mathrm{Xe}_{2}^{+}$molecular ions formed through associative ionization. Figure 2(b) presents data for blue $(\Delta>0)$ detunings of the probe laser, showing the shielding effect. Collision suppression is seen at the time when the atoms excited at $R_{c}$ by the probe laser would have collided had they continued to approach each other with their normal thermal velocity. The peak enhancement for red detunings occurs earlier than the minimum in the shielding data, showing the acceleration in the enhancement processes.

The associative ionization feature seen in the enhancement data is noticeably absent in the shielding case. As shielding affects only $6 s+6 s$ collisions, this indicates that the formation of molecular ions is due only to atom pairs which survive in and collide on the excited-state potentials (EAI). We thus observe the excited-state survival which has long been thought to play an important role in the enhancement of ultracold collisions [13-15]. Excitedstate survival in this system does not involve the population of long-lived states (such as the $1 u$ and $2 u$ states in alkali systems), which have been considered in previous work $[6,14,15]$. There is no attractive molecular potential from the $6 s+6 p$ asymptote with a lifetime more than $20 \%$ longer than the $34 \mathrm{~ns}$ atomic lifetime [16]. The states in $\mathrm{Xe}^{*}$ analogous to the long-lived alkali states are repulsive, and do not contribute to the enhancement effect.

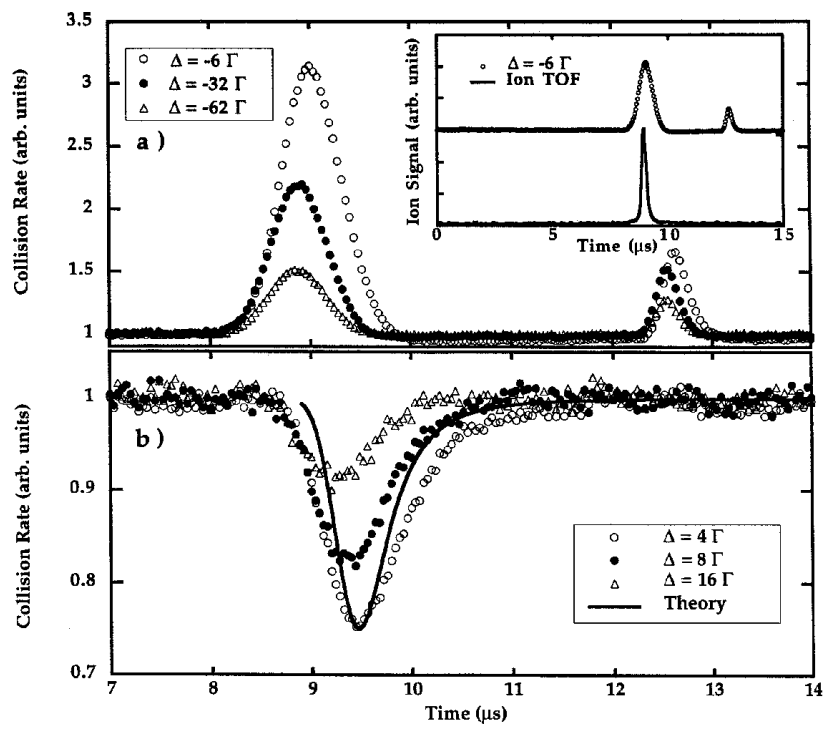

FIG. 2. Time-resolved collision signals from a $\sim 750 \mathrm{~mW} /$ $\mathrm{cm}^{2}, 40 \mathrm{~ns}$ probe pulse at $t=0$. (a) Collision enhancement for $\Delta<0(\Gamma=5 \mathrm{MHz})$. The first peak is from $\mathrm{Xe}^{+}$(Penning ionization), the second, a factor of $\sqrt{2}$ later in time, from $\mathrm{Xe}_{2}^{+}$ (associative ionization). Inset: Expanded view of the full time of flight signal, including the signal from direct photoionization of the MOT in a separate experiment (solid line), peaked at $t_{o}=8.90(5) \mu \mathrm{s}$. (b) Shielding for $\Delta>0$. The solid line is calculated for $\Delta=4 \Gamma$ using the model discussed in the text. 
As the probe is detuned farther to the red [Fig. 2(a)], the magnitude of the enhancement decreases, in a manner consistent with steady-state experiments [1]. We find that the ratio of $\mathrm{Xe}^{+}$counts $\left[N_{\mathrm{PI}}(\Delta)=N_{\mathrm{EPI}}(\Delta)+N_{\mathrm{GPI}}(\Delta)\right]$ to $\mathrm{Xe}_{2}^{+}$counts $\left[N_{\mathrm{AI}}(\Delta)=N_{\mathrm{EAI}}(\Delta)\right.$ only] decreases from greater than 10:1 at the smallest detunings used, approaching 4:1 at large $\Delta$ (Fig. 3). As we excite at larger $\Delta$ (smaller $R_{c}$ ), the survival probability for atom pairs excited to the $6 s+6 p$ potential should approach $100 \%$, as the time required to travel from $R_{c}$ to small $R$ decreases. The Penning ion counts are then predominantly due to EPI, and the ratio of $N_{\mathrm{PI}} / N_{\mathrm{AI}} \approx N_{\mathrm{EPI}} / N_{\mathrm{EAI}}$ is a measure of the relative probabilities of excited-state Penning and associative ionization. Our value of 4:1 suggests that $20 \%$ of collisions occurring in the excited state result in molecule formation [8].

The large $N_{\mathrm{PI}} / N_{\mathrm{AI}}$ ratio at small detunings is due to $6 s+6 s$ (GPI) collisions occurring through flux enhancement, which accounts for almost $60 \%$ of the total collisional enhancement $\left(N_{\mathrm{PI}}+N_{\mathrm{AI}}\right)$ at the smallest experimental detunings. This shows that the flux enhancement effect, which has only recently been observed in collision studies [3], is the dominant mechanism for laser-enhanced collisions at small detunings, and must be included in models of collisions in optical traps.

Returning to the shielding data [Fig. 2(b)], we see that as the blue detuning is increased, the effectiveness of the shielding is reduced, as seen in steady-state experiments [1]. The minimum in the collision rate (most effective shielding), for any given detuning, occurs at earlier times for larger detunings. This can be understood in our simple model of the collision process: we shield collisions by exciting pairs of atoms at a specific radius $R_{c} \propto 1 / \Delta^{1 / 3}$. Assuming a flat ground-state potential (reasonable for the $1 / R^{6} 6 s+6 s$ potential at typical $R_{c}$ ), the time required for these atoms to collide, had they not been shielded (and hence, the time required to observe the minimum collision rate), is $\tau \sim R_{c} / v_{T}$, where $v_{T}$ is the thermal velocity of the atoms.

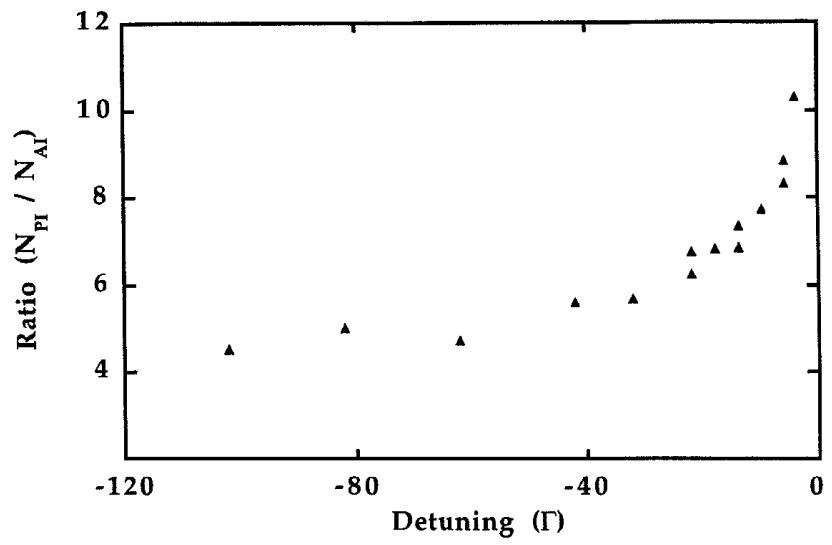

FIG. 3. The ratio of total counts in the Penning ion signal $\left(N_{\mathrm{PI}}\right)$ to total counts in the associative ionization signal $\left(N_{\mathrm{AI}}\right)$, as a function of detuning.
To model this process, we take the pair distribution function $d N(R, E, l)$, for the number of pairs in the $l$ th partial wave with separation $R$ and energy $E$, used in Ref. [17], and calculate the population and velocity distribution of atoms at the Condon radius (assuming a $\delta$-function excitation) for each of the 20 repulsive $\mathrm{Xe}^{*}$ states, calculated using theoretical values of $C_{3}[16]$. We then calculate the distribution of collision times resulting from these populations, summing the $s, p$, and $d$ partial waves $(l=0,1,2)[18]$. We average the resulting curves, weighted by $R_{c}^{2}$ to account for the number of atoms in a shell of radius $R_{c}$, and convolve the result with our experimental resolution.

The solid curve in Fig. 2(b) shows the result of such a calculation for $\Delta=4 \Gamma$ (where $\Gamma=5 \mathrm{MHz}$ is the natural linewidth), scaled to match the amplitude of the experimental signal. The general shape of the curve is well reproduced by the theory, though the theoretical curve is somewhat narrower than the data. It may be possible to improve the agreement by including excitation over a range about $R_{c}$, rather than the $\delta$-function excitation assumed here [7].

Figure 4 shows the measured collision time (referenced to the ion time of flight signal shown in Fig. 2) as a function of $1 / \Delta^{1 / 3}$ (which is proportional to $R_{c}$ ). The success of this simple model of shielding (solid line) is striking. The data fit well to a line, consistent with our picture of atoms moving together at constant velocity from the Condon radius. Comparing the slope of the fit to slopes calculated using single values of $C_{3}$ [19], we extract an effective $C_{3}$ for the shielding process, using the thermal velocity of

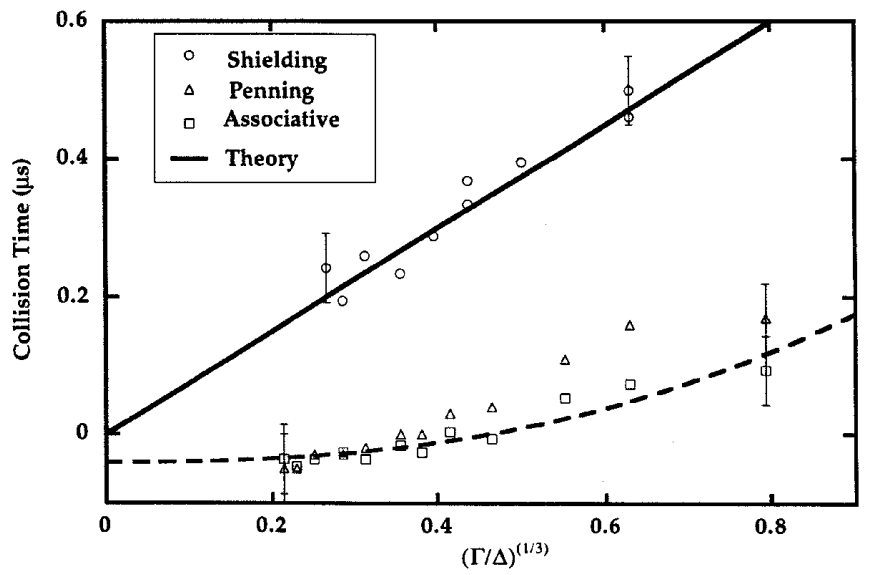

FIG. 4. Collision times $\tau$ as a function of $(1 / \Delta)^{1 / 3}\left(\propto R_{c}\right)$. Shielding and Penning ionization times are measured from the peak of the ion time of flight signal $\left(t_{o}\right)$ shown in the inset in Fig. 2 to the point of minimum or maximum collision rate. Associative ionization collision times are referenced to $\sqrt{2} t_{o}$. The error bars reflect systematic uncertainties in the measurement of peak positions. The dashed line is a fit to the associative ionization data using the survival time of Ref. [15]; the $\tau=-40 \mathrm{~ns}$ intercept is consistent with $\tau=0$. The solid line is the prediction of the simple model discussed in the text. 
$v_{T}=6.0 \mathrm{~cm} / \mathrm{s}$, and find a value of $11(3) \times\left(e a_{o}\right)^{2}[12]$ ( $e$ is the electron charge). This is in agreement with the $11.0\left(e a_{o}\right)^{2}$ average (using the same $R_{c}^{2}$ weighting function) of the theoretical $C_{3}$ values. By changing the detuning of the trapping lasers, we varied the thermal velocity by a factor of 2, and find that the collision times are linear in $1 / v_{T}$, again in agreement with our model.

Also shown in Fig. 4 are the observed collision times for enhancement of both Penning and associative ionization. The broken line is a fit to the associative ionization data using the collision time calculated from the simple model in Ref. [15]. Collision times for both processes are significantly shorter than for the shielding case, showing that acceleration on the excited-state potential is the dominant effect in determining the collision time. This is supported by the observation that both Penning and associative collision times are independent of the initial velocity $v_{T}$.

In conclusion, we use short laser pulses to study the time dependence of ionizing collision processes in detail. We observe the production of molecular ions from excited-state collisions, and estimate the rate of molecule formation from such collisions. We show that the flux enhancement effect dominates the collision enhancement process for long-range excitations, and must be included in models of collisions in optical traps. We find that conceptually simple models provide good qualitative and quantitative agreement with the data. Interesting theoretical issues remain in interpreting the details of the collision enhancement and associative ionization processes.

We thank P. Julienne and E. Tiesinga for helpful discussions. Special thanks are due to P. L. Gould for discussions which helped motivate these experiments, and to $\mathrm{M}$. Doery for calculating the molecular potentials used in our model. This work was supported in part by the U.S. Office of Naval Research. C. O. is supported by NIST/ UMCP. S. K. acknowledges the support of the Alexander von Humboldt Foundation.

* Permanent address: Chemical Physics Program, University of Maryland, College Park, MD 20742-2431.

[1] M. Walhout, U. Sterr, C. Orzel, M. Hoogerland, and S. L. Rolston, Phys. Rev. Lett. 74, 506 (1995).
[2] H. Katori and F. Shimizu, Phys. Rev. Lett. 73, 2555 (1994).

[3] V. Sanchez-Villicana, S. D. Gensemer, and P. L. Gould, Phys. Rev. A 54, R3730 (1996).

[4] L. Marcassa, S. Muniz, E. de Queiroz, S. Zilio, V. Bagnato, J. Weiner, P. S. Julienne, and K.-A. Suominen, Phys. Rev. Lett. 73, 1911 (1994); V. Bagnato, L. Marcassa, C. Tsao, Y. Wang, and J. Weiner, Phys. Rev. Lett. 70, 3225 (1993).

[5] P. D. Lett, P. S. Jessen, W. D. Phillips, S. L. Rolston, C. I. Westbrook, and P. L. Gould, Phys. Rev. Lett. 67, 2139 (1991).

[6] S. D. Gensemer and P. L. Gould, Phys. Rev. Lett. 80, 936 (1998).

[7] K.-A. Suominen, J. Phys. B 29, 5981 (1996).

[8] The relative rates of Penning and associative ionization will depend upon the molecular potentials at short range, which have not been calculated for this system.

[9] Unlike alkali systems, the ${ }^{132} \mathrm{Xe}$ isotope used for this work has no nuclear spin.

[10] M. Walhout, H. J. L. Megens, A. Witte, and S. L. Rolston, Phys. Rev. A 48, R879 (1993).

[11] Studies of the intensity dependence show that both shielding and enhancement processes begin to saturate at intensities of $\sim 400 \mathrm{~mW} / \mathrm{cm}^{2}$. This is consistent with the theory of Ref. [7].

[12] All quoted uncertainties are 1-standard deviation values.

[13] A. Gallagher and D. E. Pritchard, Phys. Rev. Lett. 63, 957 (1989).

[14] P. D. Lett, K. Molmer, S. D. Gensemer, K. Y. N. Tan, A. Kumarakrishnan, C.D. Wallace, and P.L. Gould, J. Phys. B 28, 65 (1995).

[15] K.-A. Suominen, K. Burnett, P. S. Julienne, M. Walhout, U. Sterr, C. Orzel, M. Hoogerland, and S. L. Rolston, Phys. Rev. A 53, 1678 (1996).

[16] M. Doery, E. J. D. Vredenbregt, J. G. C. Tempelaars, H. C. W. Beijerinck, and B. J. Verhaar, Phys. Rev. A 57, 3603 (1998); M. Doery (private communication).

[17] P. Julienne and J. Vigue, Phys. Rev. A 44, 4464 (1991).

[18] The $d$-wave contribution is quite small, and higher partial waves are negligible.

[19] Numerically, we find that for distributions calculated using single values of $C_{3}$, the slopes fit to plots like Fig. 4 must be multiplied by a factor of 2.8 to extract the original $C_{3}$. We apply the same correction to the fit to our data. 